\title{
COMPARAÇÃO ENTRE AS COMPETÊNCIAS DO GRAND CHALLENGES SCHOLARS PROGRAM E DAS DCNS DO CURSO DE GRADUAÇÃO DE ENGENHARIA
}

Vitória Bento Botelho - vivi.bbotelho@gmail.com

Instituto Mauá de Tecnologia, Curso de Engenharia Mecânica

Praça Mauá, 01, Bairro Mauá

09580900 - São Caetano do Sul - São Paulo

Octavio Mattasoglio Neto - omattasoglio@maua.br

Instituto Mauá de Tecnologia, Ciclo Básico

Praça Mauá, 01, Bairro Mauá

09580900 - São Caetano do Sul - São Paulo

João de Sá Brasil Lima - joao.brasil@maua.br

Instituto Mauá de Tecnologia, Engenharia Mecânica

Praça Mauá, 01, Bairro Mauá

09580900 - São Caetano do Sul - São Paulo

Resumo: Este artigo expõe e compara as competências exigidas pelas Diretrizes Nacionais Curriculares do curso de Graduação de Engenharia e pelo programa Grand Challenges Schollars Program, criado pela National Academy of Engeneering. Essa análise tem como objetivo localizar os pontos de convergência dessas competências o que pode facilitar a integração o programa citado nos cursos de uma instituição de ensino de Engenharia do Brasil. Além disso, foi abordada a origem dessas competências, assim como a sua importância no ensino como um novo modelo promissor nas instituições de ensino. Cada vez mais o mercado de trabalho aparece com novas exigências, o que demanda do engenheiro uma maior flexibilidade e rapidez ao se deparar com um problema. Essa nova abordagem por competências disponibiliza as ferramentas necessárias tanto no âmbito profissional como no pessoal para a resolução de problemas. O trabalho está baseado no método de pesquisa de análise documental, tendo como referências documentos do GCSP, das DCNs, artigos e livros. A análise feita em ambos os referenciais mostra que eles perseguem o mesmo objetivo, formar engenheiros globais, ou seja, não apenas capazes de terem ideias inovadoras, mas com a capacidade de usá-las para o avanço da sociedade como um todo. Tanto as DCNs quanto o GCSP se preocupam com a formação de um engenheiro global. Logo, é possível observar intersecção de competências desejáveis de serem atingidas $e$ isso favorece a implantação desse programa em escolas de engenharia.

Palavras-chave: Competências na engenharia. GCSP. DCNs Engenharia. 


\section{INTRODUÇÃO}

Este trabalho tem como objetivo identificar e comparar as competências das Diretrizes Curriculares Nacionais (DCNs) de engenharia com as competências do Grand Challenges Scholars Program (GCSP). A importância dessa comparação está no fato de que hoje, no Brasil, existe a necessidade de implantação das DCNs mas há escolas quem têm interesse em ingressar no GCSP, e a comparação das competências desses dois instrumentos, pode dar subsídio para a implantação de ambas.

Para isso, estudou-se o programa GCSP, um suplemento educacional criado pela National Academy of Engineering, tendo como motivação que as escolas e instituições se interessaram pelos 14 desafios do NAE Grand Challenges for Engineering, para melhorar a vida das pessoas com soluções de engenharia no século XXI. Esse programa incentiva a criação de projetos inovadores em instituições de ensino que, para participarem precisam seguir cinco competências ao abordarem o objetivo escolhido. Essas competências visam promover a formação de um engenheiro preocupado com problemas globais. Neste trabalho, as competências do GCSP foram levantadas e analisadas juntamente com as competências exigidas nas DCNs de Engenharia, atualizadas em 2019, com início em 1996 na Lei de Diretrizes e Bases da Educação LDB. O propósito desta comparação é documentar suas semelhanças e eventuais divergências.

Há que se destacar que essa comparação vai ao encontro da opção pela implantação do programa GCSP pelo Instituto Mauá de Tecnologia - IMT - que está em processo de habilitação para participar desse programa. Esta pesquisa faz parte de um projeto de Iniciação Científica que, nesta etapa, visa pesquisar a definição e a importância de competências e como elas poderão contribuir para a formação do engenheiro com a intenção de alinhar o documento nacional sobre engenharia, as DCNs, e um futuro programa de GCSP a ser implementado no IMT.

As exigências impostas ao engenheiro do século XXI, tanto profissionalmente quanto socialmente estão sofrendo mudanças em decorrência das alterações do mercado de trabalho e da sociedade. Um exemplo é o empenho em criar e melhorar processos sustentáveis, visando uma melhor qualidade de vida da população (CARVALHO \& TONINI, 2017). Portanto, desenvolver projetos que se preocupem com as competências desenvolvidas pelo aluno é importante para um melhor entendimento da formação do engenheiro do século XXI e é essencial para seu desempenho em seu futuro profissional. O capital humano é de grande importância para o desenvolvimento econômico e social e, por esse motivo, é fundamental sempre procurar evoluir na formação desse recurso humano.

\section{REFERENCIAL TEÓRICO}

Através da pesquisa em diferentes artigos foi possível observar que a ideia de competência apresenta diferentes níveis de compreensão dependendo da área em que se está estudando, mas que, no geral indica uma pessoa qualificada para realizar uma certa atividade. As competências dependem do contexto, apenas o conhecimento de um indivíduo não configura a ideia de competência a não ser que sejam comunicados e utilizados. Para a competência existir é necessária uma comunicação eficiente e para tal a rede de conhecimento em que o indivíduo se insere faz toda a diferença (FLEURY \& FLEURY, 2001). 
Atentando especificamente para a ideia de competência nos sistemas educacionais, é aceita a definição de capacidade de agir eficazmente em um determinado tipo de situação, apoiada em conhecimentos, mas sem limitar-se a eles (PERRENOUD, 1999, p.7).

As exigências impostas ao engenheiro do século XXI, tanto profissionalmente quanto socialmente estão sofrendo mudanças em decorrência das alterações do mercado de trabalho e da sociedade. Um exemplo é o empenho em criar e melhorar processos sustentáveis, visando uma melhor qualidade de vida da população (CARVALHO \& TONINI, 2017). Com essa abordagem de ensino é possível uma aprendizagem em ritmo individualizado, podendo ocorrer muitas vezes com maior velocidade do que noutra sem essa abordagem. Além disso, o aluno consegue desenvolver as habilidades exigidas pelo mercado de trabalho durante o curso.

A forma de conduzir bem a vida social e profissional dependem da capacidade do indivíduo de resolver problemas cotidianos. Dessa forma, é possível perceber que uma competência não pode ser transmitida, mas sim desenvolvida.

\subsection{Competências nas DCNs}

As DCNs têm origem na Lei de Diretrizes e Bases da Educação (LDB), de 1996, que assinala ser incumbência da União,

estabelecer, em colaboração com os estados, Distrito Federal e os municípios, competências e diretrizes para a Educação Infantil, o Ensino Fundamental e o Ensino Médio, que nortearão os currículos e os seus conteúdos mínimos, de modo a assegurar a formação básica comum.

A seguir estão listadas as oito competências indicadas nas DCNs de engenharia (ABENGE, 2019) homologadas em 2019.

I - formular e conceber soluções desejáveis de engenharia, analisando e compreendendo os usuários dessas soluções e seu contexto: a) ser capaz de utilizar técnicas adequadas de observação, compreensão, registro e análise das necessidades dos usuários e de seus contextos sociais, culturais, legais, ambientais e econômicos; b) formular, de maneira ampla e sistêmica, questões de engenharia, considerando o usuário e seu contexto, concebendo soluções criativas, bem como o uso de técnicas adequadas;

II - analisar e compreender os fenômenos físicos e químicos por meio de modelos simbólicos, físicos e outros, verificados e validados por experimentação:

a) ser capaz de modelar os fenômenos, os sistemas físicos e químicos, utilizando as ferramentas matemáticas, estatísticas, computacionais e de simulação, entre outras.

b) prever os resultados dos sistemas por meio dos modelos;

c) conceber experimentos que gerem resultados reais para o comportamento dos fenômenos e sistemas em estudo.

d) verificar e validar os modelos por meio de técnicas adequadas;

III - conceber, projetar e analisar sistemas, produtos (bens e serviços), componentes ou processos:

a) ser capaz de conceber e projetar soluções criativas, desejáveis e viáveis, técnica e economicamente, nos contextos em que serão aplicadas;

b) projetar e determinar os parâmetros construtivos e operacionais para as soluções de Engenharia;

c) aplicar conceitos de gestão para planejar, supervisionar, elaborar e coordenar projetos e serviços de Engenharia;

IV - implantar, supervisionar e controlar as soluções de Engenharia:

a) ser capaz de aplicar os conceitos de gestão para planejar, supervisionar, elaborar e coordenar a implantação das soluções de Engenharia. 
b) estar apto a gerir, tanto a força de trabalho quanto os recursos físicos, no que diz respeito aos materiais e à informação;

c) desenvolver sensibilidade global nas organizações;

d) projetar e desenvolver novas estruturas empreendedoras e soluções inovadoras para os problemas;

e) realizar a avaliação crítico-reflexiva dos impactos das soluções de Engenharia nos contextos social, legal, econômico e ambiental;

V - comunicar-se eficazmente nas formas escrita, oral e gráfica:

a) ser capaz de expressar-se adequadamente, seja na língua pátria ou em idioma diferente do Português, inclusive por meio do uso consistente das tecnologias digitais de informação e comunicação (TDICs), mantendo-se sempre atualizado em termos de métodos e tecnologias disponíveis;

VI - trabalhar e liderar equipes multidisciplinares:

a) ser capaz de interagir com as diferentes culturas, mediante o trabalho em equipes presenciais ou a distância, de modo que facilite a construção coletiva;

b) atuar, de forma colaborativa, ética e profissional em equipes multidisciplinares, tanto localmente quanto em rede;

c) gerenciar projetos e liderar, de forma proativa e colaborativa, definindo as estratégias e construindo o consenso nos grupos; PROCESSO Nº: 23001.000141/2015-11 Antonio Freitas e outros -014138

d) reconhecer e conviver com as diferenças socioculturais nos mais diversos níveis em todos os contextos em que atua (globais/locais); e) preparar-se para liderar empreendimentos em todos os seus aspectos de produção, de finanças, de pessoal e de mercado;

VII - conhecer e aplicar com ética a legislação e os atos normativos no âmbito do exercício da profissão:

a) ser capaz de compreender a legislação, a ética e a responsabilidade profissional e avaliar os impactos das atividades de Engenharia na sociedade e no meio ambiente.

b) atuar sempre respeitando a legislação, e com ética em todas as atividades, zelando para que isto ocorra também no contexto em que estiver atuando; e

VIII - aprender de forma autônoma e lidar com situações e contextos complexos, atualizando-se em relação aos avanços da ciência, da tecnologia e aos desafios da inovação:

a) ser capaz de assumir atitude investigativa e autônoma, com vistas à aprendizagem contínua, à produção de novos conhecimentos e ao desenvolvimento de novas tecnologias. b) aprender a aprender. Parágrafo único. Além das competências gerais, devem ser agregadas as competências específicas de acordo com a habilitação ou com a ênfase do curso.

\subsection{Competências do GCSP}

O National Academy of Engeneering (NAE) criou em 2008 o NAE Grand Challenges for Engeneering, com o objetivo de apresentar uma visão aspiracional do que a engenharia precisa oferecer a todas as pessoas do planeta no século XXI. Essa ideia foi baseada em 14 objetivos que o NAE levantou como necessários para concretizar essa visão no século XXI considerando suas quatro grandes áreas: sustentabilidade, saúde, segurança e joy of living.

Muitas escolas de engenharia e programas de ensino fundamental e médio adotaram o NAE Grand Challenges para inspirar projetos práticos para seus alunos por meio de um suplemento educacional chamado Grand Challenges Scholars Program (GCSP). O GCSP aponta as cinco competências a seguir como essenciais para os alunos terem as ferramentas necessárias para lidar com esses desafios globais: 
I. Competência em talentos: pesquisa orientada / experiência criativa em um tópico semelhante ao Grande Desafio

II . Competência multidisciplinar: compreensão da multidisciplinaridade das soluções de sistemas de engenharia desenvolvidas por meio do engajamento pessoal

III. Competência viável de negócios / empreendedorismo: entendimento, de preferência desenvolvido por meio da experiência, da necessidade de um modelo de negócios viável para a implementação da solução

IV. Competência multicultural: entender diferentes culturas, de preferência através de experiências multiculturais, para garantir a aceitação cultural das soluções de engenharia propostas

V. Competência em Consciência Social: compreendendo que as soluções de engenharia devem servir principalmente as pessoas e a sociedade, refletindo a consciência social

\section{MÉTODO DE PESQUISA}

O método de pesquisa escolhido para a realização deste trabalho de iniciação científica foi o de análise documental. Essa técnica busca apontar informações de veracidade constatada em documentos a partir de questionamentos e hipóteses de interesse da pesquisa a ser realizada (CAULLEY, 1983). Além disso, apresenta importância ímpar na abordagem de dados qualitativos enriquecendo as informações obtidas por outras técnicas e desvendando novas faces de um tema ou problema. Contudo, destaca a exigência do rigor intelectual e dedicação, sendo dever do autor reunir os dados, estabelecer relações e associações para então combinálos, separá-los e reorganizá-los (LUDKE \& ANDRÉ, 1986).

Para esse projeto foram levantadas as competências das DCNs de engenharia, atualizadas em 2019, junto com as cinco competências requeridas pelo programa GCSP. Ambas foram analisadas e comparadas para localizar seus pontos de encontro e divergência. Construiu-se um quadro comparativo que será apresentado e discutido nos próximos itens.

A partir da referência de competências do GCSP o que se fez foi identificar nas oito competências das DCNs para os cursos de engenharia e suas subdivisões, em qual das competências do GCSP elas se encaixavam por afinidade de propósito.

\section{DADOS E RESULTADOS}

\subsection{Dados}

As competências das DCNS se preocupam com o processo de aprendizagem, ou seja, em proporcionar ferramentas para o aluno desenvolver as habilidades e competências necessárias para sua vida profissional como engenheiro. As competências do GCSP estão focadas em formar engenheiros que se preocupem com a continuidade de vida no planeta e com a qualidade da mesma. O processo do GCSP ocorre com a abordagem de problemas, criação de produtos e/ou empresas, enquanto as DCNs proporcionam a fundação do curso de engenharia. Desse modo, ambas têm a mesma intenção de melhorar a forma como é abordada a engenharia tanto em seu ensino quanto na sua parte de desenvolver novas ideias, mas não possuem o mesmo foco. Analisando-as pode-se perceber similaridades nas ideias.

A Figura 1 traz o quadro com o cruzamento entre as competências indicadas nas DCNs, e suas subdivisões, e aquelas indicadas no GCSP. 


\section{COBENCE C. COBENCE $2020 \begin{aligned} & \text { de Educação em Engenharia } \\ & \text { da ABENGE }\end{aligned}$}

Figura 1 - Comparação das competências do GCSP e das DCNs

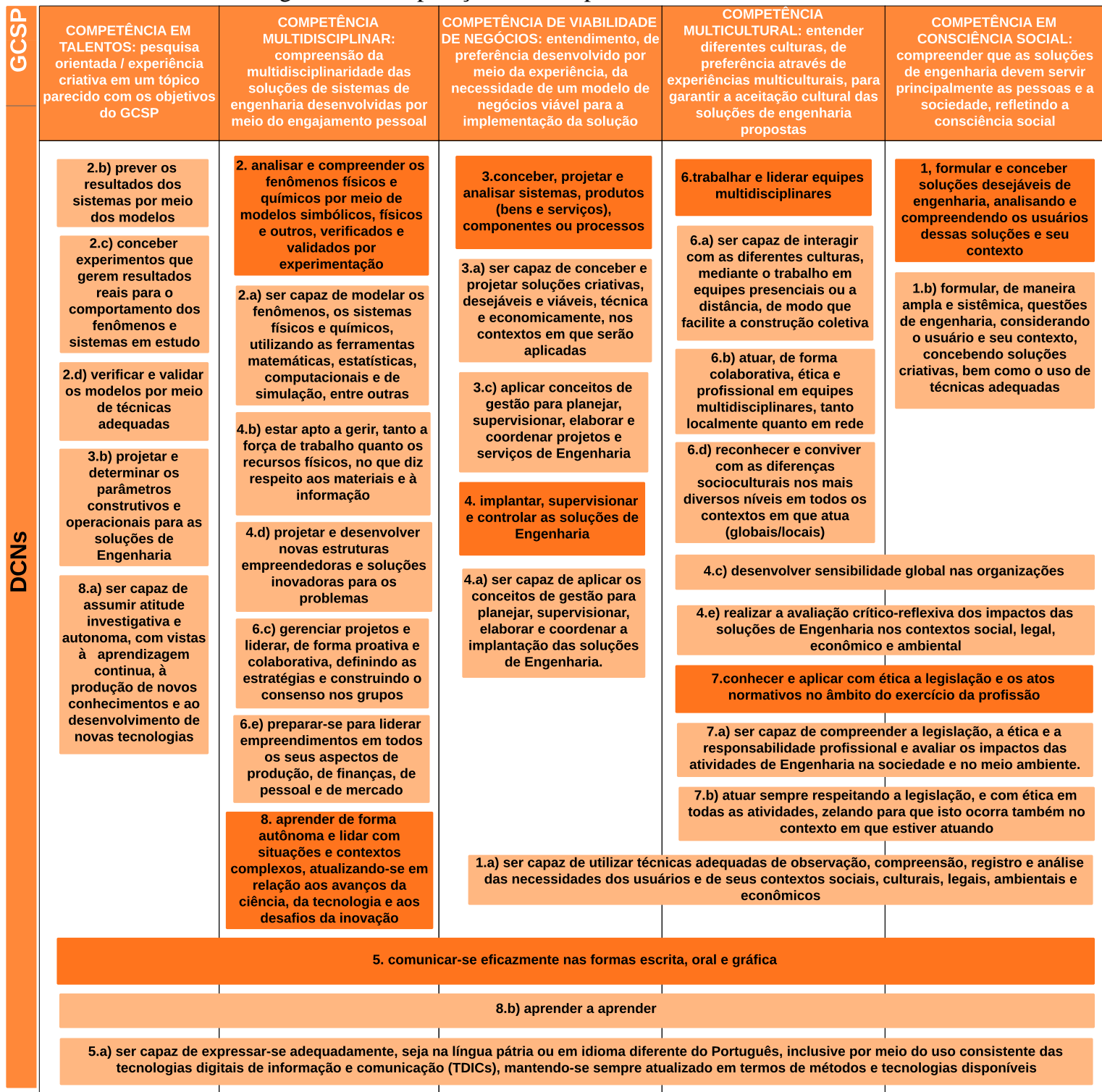

Legenda: Oito DCNs (laranja escuro), subdivisões de cada DCN (laranja claro).

Fonte: os autores.

\subsection{Discussão}

Na figura 1 foram comparadas as cinco competências do GCSP com as oito competências das DCNs. Porém, em cada competência das DCNs também foram indicadas suas subdivisões que podem variar de "a" até "e".

$\mathrm{Na}$ primeira coluna é possível observar que a Competência em Talentos do GCSP relaciona-se com as DCNs 2.b, 2.c, 2.d, 3.b e 8.a. Essa primeira competência do GCSP é sobre a capacidade de realizar uma pesquisa orientada como Iniciação Científica. No caso, essa pesquisa seria com o foco em desenvolver uma possível solução para um dos tópicos do programa, ou seja, soluções para melhorar as condições no mundo. Pode-se entender que as quatro competências das DCNs listadas acima se relacionam com esse tema do GCSP, já que tratam de habilidade necessárias para desenvolver qualquer tipo de pesquisa que almeje chegar em um resultado satisfatório. Nessa dimensão há o propósito de promover no estudante o espírito de investigação o que se traduz nas DCNs como conceber experimentos, prever resultados, verificar e validar modelos, projetar e determinar os parâmetros construtivos e 
assumir atitude investigativa. Além disso, pode-se incluir na investigação a competência de se comunicar e fazer uso da Tecnologia da Informação e Comunicação. Há portanto muitos pontos de relacionamento entre as DCNs e a competência de desenvolvimento de talentos do GCSP.

Na segunda coluna é possível observar que a Competência Multidisciplinar, que se refere a necessidade de articular várias áreas do conhecimento do engenheiro, principalmente a ciência e a tecnologia, para a resolução de um problema, relaciona-se com as DCNs 2, 2.a,4.b, 4.d, 6.c, 6.e e 8. Isso ocorre pelo fato dessas quatro competências selecionadas das DCNs tratarem da necessidade que engenheiro tem em conhecer as diversas ferramentas ao seu dispor na resolução de um problema. Em qualquer área de atuação do engenheiro são necessárias diferentes habilidades para a criação de um projeto ou para a melhoria do mesmo. Além de requisitos técnicos como analisar, compreender e modelar fenômenos físicos, o que envolve o conhecimento técnico de diversas áreas, esse profissional não pode deixar de adquirir habilidades sócio-emocionais, ou seja, precisa saber liderar equipes (6c e 6e) e liderar empreendimentos com foco inovador $(4 \mathrm{~d})$, se relacionar bem com colegas, sócios, funcionários e chefes envolvidos na sua atuação. Para completar o profissional tem que ter competência para aprender de forma autônoma e aqui se entende em diversas áreas, além da competência de comunicação. O GCSP coloca essa competência como modo de enfatizar a ideia de que sem habilidades sociais, o engenheiro terá dificuldades em aplicar suas habilidades técnicas.

$\mathrm{Na}$ terceira coluna é possível observar que a Competência em Viabilidade de Negócios do GCSP relaciona-se com as DCNs 1.a, 3, 3.a, 3.c, 4, 4.a. Essa competência exige do aluno a capacidade de definir como seria o melhor modelo de negócio para a implementação de um serviço/produto desenvolvido. Essas competências das DCNs se encaixam com a do GCSP, uma vez que todas dissertam sobre habilidades necessárias para a conceber, analisar e projetar sistemas, produtos e processos, desde a criação desse modelo até a melhor forma de liderança do mesmo, implantando, controlando e supervisionando as soluções de Engenharia. Tudo isso com foco na "observação, compreensão, registro e análise das necessidades dos usuários e de seus contextos sociais, culturais, legais, ambientais e econômicos".

$\mathrm{Na}$ quarta coluna é possível observar a Competência Multicultural do GCSP relaciona-se com as DCNs 1.a, 4.e, 4.c, 6, 6.a, 6.b, 6.d, 7, 7.a, 7.b. Essa competência refere-se a capacidade de entender e se relacionar bem com diferentes culturas para garantir a aceitação de algum projeto proposto. Essas competências das DCNs possuem relação, já que exigem essa aceitação cultural e falam sobre ferramentas para alcançar esse entendimento da melhor forma, como pelo conhecimento legislação e do contexto social da cultura/localidade onde será efetuado um possível projeto. Em uma instituição de ensino essas competências podem ser aplicadas na prática por meio de intercâmbios com instituições parceiras, pois dessa forma o aluno pode ter contato direto com diferentes culturas e aprender por meio da própria experiência. Há a indicação da necessidade de internacionalização dos cursos de engenharia, o que, nas DCNs aparece como reconhecer e conviver com as diferenças multiculturais.

Na quinta coluna é possível observar que a Competência em Consciência Social do GCSP relaciona-se com as DCNs 1, 1.a, 1.b, 4.c, 4.e, 7, 7.a, 7.b. Essa competência é similiar a Multicultural, com a diferença de não exigir um entendimento cultural, mas também exigir a compreensão que a engenharia e sua soluções devem servir principalmente a sociedade. Dessa forma, essas competências das DCNs dialogam com a do GCSP, uma vez que, falam sobre como atingir essa exigência.

Há três competências das DCNs, 5, 5.a e 8.b, que englobam todas as competências do GCSP. No caso da 5 e 5.a, ambas dissertam sobre a habilidade de se expressar, na forma oral, escrita e gráfica, a qual é necessária para desenvolver todas as competências do GCSP. A DCN 8.b é 'aprender a aprender', o que significa ter uma postura ativa na aprendizagem, apropriandose de um conteúdo, para assim assumir a gerência de seu ensino. Dessa forma, a DCN 8.b ( 
abrange todas competências desse programa internacional, uma vez que, ter uma postura ativa na aprendizagem é o próprio meio de adquirir qualquer competência.

\section{CONCLUSÃO}

O ensino por competências é um novo referencial na construção pessoal e profissional do engenheiro global, como citado anteriormente nesta pesquisa. O GCSP e as DCNs aceitam e incentivam essa abordagem, por isso a importância de comparar suas competências e mostrar seus pontos convergentes e divergentes, facilitando a implantação do GCSP em escolas de engenharia.

A análise feita em ambos mostra que eles perseguem o mesmo objetivo, formar engenheiros não apenas capazes de ter ideias inovadoras, mas com a capacidade de usá-las para o avanço da sociedade como um todo, utilizando principalmente da experiência como método educativo, contudo, elas possuem focos diferentes. As DCNs tratam da estruturação do curso de engenharia, alinhando todas as instituições de engenharia para garantir uma boa formação ao engenheiro. Dessa forma, as diretrizes precisam ser mais genéricas e abrangentes para atingir os requisitos de todas as áreas da engenharia e, também, de qualquer escolha profissional do engenheiro formado. O GCSP é um programa suplementar que tem como foco quatro grandes áreas: saúde, segurança, sustentabilidade e joy of living. A intenção é agregar para a formação do engenheiro que, por sua vez, já deve estar alinhado com as DCNs, já que o GCSP é um programa suplementar, então não é responsável por si só para a formação desse profissional. A contribuição desse programa suplementar é fazer com que esse engenheiro tenha uma visão global, assim, poderá utilizar o seu conhecimento para ajudar o mundo como um todo e não apenas a sua comunidade. Ambos tratam de dimensões relacionadas à construção da aprendizagem do aluno e se atentam em colocar o foco no método de ensino por competências na forma de vivências, o que leva o aluno engenheiro questionar o que é possível fazer com todo seu conhecimento adquirido. A instituição de ensino pode escolher, dentro dos requisitos do GCSP, a melhor forma de inserir esse programa, não sendo necessária uma mudança curricular da instituição.

Os ideais específicos definidos nas competências do no GCSP encontram-se diluídos nas DCNs. Incluir o GCSP em uma instituição de ensino pode levar a criação de uma competência suplementar, que é a de formação do engenheiro cidadão do mundo, tal como declarado no propósito do GCSP.

Atender o GCSP e atender as DCNs não são caminhos diversos, ao contrário, a intersecção entre essas abordagens indica que atender as DCNs para os cursos de engenharia coloca as escolas em condição técnica de atender o GCSP, isso é um fator encorajador para participar do GCSP. No entanto, o GCSP não é apenas uma diretriz técnica há um propósito que é a criação de uma identidade profissional que somente se alcança com o envolvimento efetivo do estudante com desafios globais, o que exige um envolvimento e empatia com os problemas que parece somente podem ser alcançados com um percurso que permita efetivamente $o$ desenvolvimento de competências que tornem esse profissional sensível aos problemas globais.

\section{Agradecimentos}

Ao Instituto Mauá de Tecnologia pelo apoio na forma de bolsa de Iniciação Científica.

\section{REFERÊNCIAS}




\section{COBENCE 2020 de Educação em Engenharia de Educação em Engenharia da ABENGE}

ABENGE - Proposta de parecer e de resolução para as DCNs Engenharia. http://www.abenge.org.br/file/Minuta\%20Parecer\%20DCNs_07\%2003\%202018.pdf Accessed 04 March 2019.

CARVALHO, L. de A.; TONINI, A. M. Uma análise comparativa entre as competências requeridas na atuação profissional do engenheiro contemporâneo e aquelas previstas nas diretrizes curriculares nacionais dos cursos de Engenharia. São Carlos, v. 24, ed. 4, p. 829$841,2017$.

CAULlEY, D. N. Document Analysis in Program Evaluation. La Trobe University, 1983.

LUDKE, M.; E. D. A. ANDRÉ, M. Pesquisa em educação: abordagens qualitativas. São Paulo, 1986

MEC - Ministério da Educação e Cultura. Conselho Nacional de Educação. Câmara de Educação Superior. Diretrizes Curriculares para os cursos de engenharia. Resolução CNE/CES nº 2, de 24 de abril de 2019.

MEC - Ministério da Educação e Cultura. Conselho Nacional de Educação. Câmara Nacional de Educação Básica. Diretrizes Curriculares Nacionais Gerais da Educação Básica. MEC, SEB, DICEI, 2013.

MESQUITA, D., LIMA, R. M., \& FLORES, M. A. Developing professional competencies through projects in interaction with companies: A study in Industrial Engineering and Management Master Degree. Proceedings: Fifth International Symposium on Project Approaches in Engineering Education, PAEE'2013. Eindhoven, The Netherlands. 2013. Actas: PAEE'2013. Eindhoven, The Netherlands. 2013.

MESQUITA, D., LIMA, R. M., FLORES, FLORES, M. A. Developing professional competencies through projects in interaction with companies: A study in Industrial Engineering and Management Master Degree. $5^{\text {th }}$ International Symposium on Project Approaches in Engineering Education, PAEE'2013. Eindhoven, The Netherlands. 2013.

NATIONAL ACADEMY OF ENGINEERING (Estados Unidos). Grand Challenges Schollars Program (org.). NAE Grand Challenges Scholars Program: Grand Challenges Scholars Program. Estados Unidos: National Academy of Engineering, [200-].

PERRENOUD, P. Construir as competências desde a escola. Porto Alegre, Artmed, 1999.

TEREZA L. FLEURY, M; FLEURY, A. Construindo o conceito de competência. Curitiba, 2001.

COMPARISON BETWEEN THE COMPETENCES OF THE GRAND CHALLENGES SCHOLARS PROGRAM AND THE DCNS OF THE ENGINEERING GRADUATION PROGRAM 


\begin{abstract}
This work exposes and compares the competences required by the National Curriculum Guidelines for the Undergraduate Engineering course and the Grand Challenges Schollars Program, created by the National Academy of Engineering. This analysis aims to locate the points of convergence or divergence of these competences in order to integrate the program mentioned in the courses of an academic institution. In addition, the origin of these competences was addressed, as well as their importance in teaching as a promising new model in educational institutions. Increasingly, the job market appears with new requirements, which demands from the engineer greater flexibility and speed when faced with a problem. This new competency-based approach provides the tools needed both professionally and personally for problem solving. The work is based on the documental analysis research method, with articles and books as references. The analysis made in both references shows that they pursue the same objective, to build global engineers, that is, not only capable of having innovative ideas, but with the ability to use them for the advancement of society as a whole. While the DCNs have a main focus on the structuring of a course, the GCSP pursues the formation of a global engineer, but, mainly, there is a great intersection of competences that is desirable to achieve in these references, which favors the implantation of this program in engineering schools. Both the DCNs and the GCSP are concerned with building a global engineer. Therefore, it is possible to observe the intersection of desirable skills to be achieved and this favors the implementation of this program in engineering schools.
\end{abstract}

Keywords: Engineering competences. GCSP. Engineering DCNs. 